\title{
Does Etanercept Biosimilar Prescription in a Rheumatology Center Bend the Medication Cost Curve?
}

\author{
Wieland D. Müskens ${ }^{1}$ (D), Sanne A.A. Rongen-van Dartel² ${ }^{\text {DD }}$, Piet L.C.M. van Riel² (D), \\ and Eddy M.M. Adang ${ }^{3}$ (D)
}

\begin{abstract}
Objective. The market entry of biosimilars is expected to bring budgetary relief. Our objective was to determine how the introduction of biosimilars influences medication costs in patients with rheumatoid arthritis (RA) and which patients gain access to biologics due to the availability of biosimilars.

Methods. Using hospital data of patients with RA between 2014 and 2018, an interrupted time series was performed. The interruption in the time series was placed at June 2016 (i.e., the introduction of the etanercept biosimilar). The changes in trends for rheumatic medication costs before and after the interruption were measured. Secondary analyses focused on explaining these trends.

Results. In the first quarter after the interruption, there was a decrease in total costs for biologic users of -€63,020 (95\% CI -€96,487 to -€29,553, P = 0.001). The postinterruption trend did not differ from the preinterruption trend ( $95 \% \mathrm{CI}-€ 6695$ to $€ 6715, P=0.998$ ) and after 3 quarters, the medication costs were back at the interruption level. After the interruption, the average cost per biologic user decreased by $-€ 370$ (95\% CI $-€ 602$ to $-€ 138, P=0.005$ ), followed by a quarterly decrease (relative to the preinterruption trend; $95 \% \mathrm{CI}-€ 86$ to $-€ 14, P=0.010$ ), bending the average cost curve. The percentage of patients being treated with biologics increased in postinterruption by 0.50 percentage points quarterly ( $95 \% \mathrm{CI} 0.38-0.62$, $P<0.001)$. Also, the average age at the start of the first biologic increased after the interruption $(P=0.057)$. Conclusion. The average cost per patient treated with biologics decreased after the introduction of biosimilars with a persistent trend. However, the budgetary relief due to market entry of biosimilars vanished quickly due to an increase in patients treated with biologics.
\end{abstract}

Key Indexing Terms: biologic therapy, biosimilars, cost savings, rheumatic diseases

Cost containment in health care is a major issue in Western countries. However, healthcare expenditures continue to grow. It is generally believed that when a patent on pharmaceuticals expires, this leads to a drop in healthcare costs. Whether and how this actually occurs in the Netherlands is investigated in this paper. All expensive medication in the Netherlands is financed

This work was supported by a grant from Stichting RUN. The opinions, results, and conclusions reported in this paper are those of the authors and are independent from the funding sources. No endorsement by Stichting RUN is intended or should be inferred.

${ }^{I}$ W.D. Müskens, $M D, M S c$, Department of IQ Healthcare, Radboudumc, Nijmegen; ${ }^{2}$ S.A. Rongen-van Dartel, PhD, P.L. van Riel, MD, PhD,

Department of IQ Healthcare, Radboudumc, Nijmegen, and Department of Rheumatology, Bernhoven, Uden; ${ }^{3}$ E.M. Adang, PhD, Department of Health Evidence, Radboudumc, Nijmegen, the Netherlands.

$P L v R$ has received grant funding from the Rheumatoid Arthritis Foundation to support this work, and has received speaker fees or grants from AbbVie, Eli Lilly, Pfizer, and UCB in the past 3 years. WDM was undertaking an academic promotion during the time of the study. The remaining authors have no other relationships or activities that could appear to have influenced the submitted work to disclose.

Address correspondence to Dr. W.D. Müskens, Radboudumc, Radboud Institute for Health Sciences, IQ Healthcare, P.O. Box 9101, $6500 \mathrm{HB}$ Nijmegen, the Netherlands.Email: wieland.muskens@radboudumc.nl. Accepted for publication October 21, 2020. through the hospital system, and costs are therefore part of Medical Specialist Care (MSC). However, at this point, the growth of expensive medication costs exceeds the growth rate of the MSC as a whole. This means, given a fixed budget, that the expenditure on expensive medicines is displacing other care. ${ }^{1}$

The focus of the present study is on biologic medication of which the patent period has expired. It is anticipated that a decrease in prices for expensive medication, initiated through the availability of unpatented biologics, known as biosimilars, will lead to lower total costs and create budgetary relief. ${ }^{1}$ Biosimilars are biotherapeutic products (biologics) that are similar in terms of quality, safety, and efficacy to an already licensed reference biotherapeutic product (bio-originator). ${ }^{2}$ However, biosimilars have a significantly lower price and thereby induce price competition between bio-originator and biosimilar producers, which is assumed to result in cost savings and finally bend the (total) cost curve.

These expectations regarding the predicted budget effect of biosimilars are based on many studies performed in the build-up to patent expiration of bio-originators. ${ }^{3,4,5,6,7,8}$ All of these studies predicted a cost savings. The amount of cost savings predicted differed, depending on the acquisition cost of the biosimilar drug, ${ }^{8}$ the initial number of patients being treated with biologic therapy, ${ }^{8}$ the number of biosimilars available, ${ }^{6}$ and the uptake of biosimilar use. $^{5}$

One of the fields where the introduction of biosimilars is 
predicted to generate savings is in the treatment of rheumatoid arthritis (RA)., ${ }^{5,8}$ At this point, biosimilars for the tumor necrosis factor (TNF)- $\alpha$ blockers (a subgroup of biologics) adalimumab (ADA), etanercept (ETN), and infliximab (IFX) have been approved by the European Medicines Agency. ${ }^{9}$ The expectations for biosimilars with regard to cost savings are high. For example, the chief executive officer of the National Health Service (NHS) previously announced that he expects the use of an ADA biosimilar to free up $£ 300$ million ( $€ 340$ million) in the United Kingdom, which can be invested in patient care in general. ${ }^{10}$ To assess the real-world effect of biosimilars, the effect of IFX and ETN biosimilars on the biologic disease-modifying antirheumatic drug (bDMARD) budget in the UK was studied. ${ }^{11}$ The main finding of that study was that the introduction of biosimilars indeed resulted in lower medication prices due to price reduction of both the bio-originators and the biosimilars. However, their data also showed an increase in the overall utilization of biologics, although they did not explain this finding further. Their data showed that this increased utilization of bDMARDs outweighed the price reduction achieved through the introduction of biosimilars. Therefore, no net savings were achieved even though prices for bDMARDs dropped.

Similar observations were made in the Netherlands, in a previous report based on real-world data from the Dutch Healthcare Authority (NZa). They observed a similar reduction in individual prices for existing expensive medication, accompanied with an increase in utilization of these expensive medications. Therefore the total cost for these medications increased. ${ }^{1}$ For TNF- $\alpha$ blockers specifically, they observed an increase of $11 \%$ in the volume of patients using bDMARDs. ${ }^{1}$

Both the NZa report ${ }^{1}$ and the UK study ${ }^{11}$ show an increase in the volume of bDMARDs for the treatment of RA. However, because these studies were based on national declaration data, they did not report on the mechanisms behind it nor on which patients gained access to bDMARDs. This evokes several questions from the perspective of a care provider. Is there a change in the percentage of patients using bDMARD therapy after the introduction of biosimilars? Are demographic and medical characteristics of patients receiving bDMARD treatment able to explain the potential change over time?

Our main aim was to study the effect of market entry of an ETN biosimilar on medication costs for biologic users of the RA population in a general hospital in the Netherlands. Secondary objectives were aimed at explaining the trends found.

\section{METHODS}

Design. We studied the price effect of the introduction of biosimilars in RA using a single-center interrupted time series design. The trends of total medication costs in bDMARD users and medication cost per patient using bDMARDs were compared before and after the introduction of an ETN biosimilar at the Department of Rheumatology at Bernhoven in June 2016. Bernhoven is a medium-sized hospital in the south of the Netherlands that serves as a secondary referral center in the region. This gives Bernhoven a case mix of patients that is representative for general hospitals in the Netherlands.

From June 2016 onwards, all patients who initiated ETN treatment were treated with the biosimilar. The criteria for eligibility for bDMARD treatment in the Netherlands did not change during the study period: a Disease Activity Score in 28 joints (DAS28) > 3.2 after treatment failure with at least 2 conventional synthetic DMARDs (csDMARDs), including methotrexate in a dose of $25 \mathrm{mg} /$ week. $^{12}$ Since transitioning to a biosimilar is allowed in the Netherlands, all patients with inflammatory rheumatic diseases treated with the ETN originator in Bernhoven were invited to transition to the biosimilar. Approximately $87 \%$ of these patients accepted the transition, with a 1 -year retention rate of $72 \% .^{13}$ These rates fall well within the range observed by other studies in the Netherlands. ${ }^{13}$

During the study period, an IFX biosimilar became available at the hospital. However, as the prescription of IFX for the treatment of RA is low in the Netherlands in general, only 10 patients received treatment with IFX during the study period in Bernhoven, (i.e., $<4 \%$ of the DMARD users). Therefore, we deemed the influence of the introduction of the IFX biosimilar negligible and focused on the introduction of the ETN biosimilar.

Ethics. All patients gave their informed consent for use of their medical data for scientific purpose at an earlier point in time. Ethical approval was not necessary for this study, given the registry of common practice care-based data collection.

Inclusion criteria. All patients with RA being treated at Bernhoven from June 1, 2014, until June 1, 2018, were included in the analysis, providing a representative sample for the Dutch RA population. All patients included had been diagnosed with RA by their rheumatologist according to the American College of Rheumatology 2010 criteria. $^{14}$

Data and instruments. Data on medication use of all patients with RA were collected using the electronic medical record system of Bernhoven. Information regarding the specific use of the ETN bio-originator or the ETN biosimilar was verified by the pharmacy of Bernhoven.

The following demographic and medical characteristics were collected: age, sex, rheumatoid factor (RF) positivity, anticyclic citrullinated peptide (anti-CCP) positivity, disease duration, and disease activity. The DAS28 was used as a measure of disease activity (score between 0 and 10; higher score indicates higher disease activity)..$^{15}$

The quarters followed a 3-monthly sequence starting on June 1, 2014. For each quarter, the sample consisted of all patients with an active diagnosis of RA at the department. Patients were categorized as a bDMARD user if they were treated with a bDMARD during that quarter.

Rheumatic medication cost. Rheumatic medication cost (RMC) was defined as the cost for nonsteroidal antiinflammatory drugs, csDMARDs, bDMARDs, and glucocorticoids (GCs).

In the Netherlands, bDMARDs are paid for through the hospital budget, and prices are negotiated with the pharmaceutical company per hospital. Therefore, information on the price of all bDMARDs was obtained from the pharmacy of Bernhoven to account for the negotiating bonus. These prices are confidential and therefore not disclosed here. During the study period, the hospital negotiated a discount for the ETN biosimilar.

Other medication is directly reimbursed by health insurance companies. These prices are collected through National Healthcare Institute's drug cost database (www.medicijnkosten.nl), as recommended by the Dutch guideline for cost-effectiveness research. ${ }^{16}$

Analyses. A single-center interrupted time series analysis is used that estimates the coefficients by ordinary least squares regression with Newey-West standard errors to handle autocorrelation in addition to possible heteroscedasticity in the data. In general the regression model assumes the following form:

$$
R M C_{t}=\beta_{0}+\beta_{1} T_{t}+\beta_{2} X_{t}+\beta_{3} X_{t} T_{t}+\epsilon_{t}
$$

$R M C_{t}$ is the aggregated RMC variable measured at each quarter $t$ since June 1, 2014; $T_{t}$ is the time since the start of the study; $X_{t}$ is a dummy variable for the moment of interruption; and $X_{t} T_{t}$ is an interaction term. Of interest are $\beta_{2}$ and $\beta_{3}$, which, respectively, show the immediate price effect at time of interruption and the difference in trend between pre- and postinterruption. ${ }^{17}$ Autocorrelation in the error distribution is tested by the Cumby-Huizinga general test for autocorrelation. Depending on the outcome of this test, 1 or 
more lags are added to the model above. The interruption in the time series (quarterly intervals) was placed at June 1, 2016 (i.e., the drop in price due to the introduction of biosimilars). Time series were run to assess the RMC, first in the total RA population, then in bDMARD users only. Demographic and medical variables were added to the model to explain the trends found. The total RMC of the total RA population was adjusted for bDMARD cost to study the cost of the other rheumatic medication (i.e., csDMARDs and GCs). In a separate analysis, the average cost per patient in bDMARD users was adjusted for the bDMARD dosage, to assess whether bDMARD dosage influenced the average RMC per patient. The bDMARD dosage was standardized as percentage of the daily defined dosage. ${ }^{18}$ An additional time series was run to assess the percentage of biologic users, instead of the RMC, in the RA population over time. To take into account possible demographic differences between patients using bDMARDs in the preinterruption and postinterruption period, the RMC models were run with the following covariates: BMI, age, and sex (adjusted model I). Similarly, it was investigated whether RA-specific disease variables differed between patients using bDMARDs in the pre- and postinterruption period, including RF positivity, anti-CCP positivity, age at diagnosis, age at start of first biologic, and DAS28 at start of first biologic (adjusted model II). Analyses were done in Stata 15.1 (StataCorp).

\section{RESULTS}

Patient characteristics of the RApopulation. Between June 1, 2014, and June 1, 2018, the RA population in Bernhoven increased from 640 to 961 patients. The patient characteristics of the RA population and subgroup of bDMARD users on June 1, 2016, are shown in Table 1 . At that moment, $17 \%$ of the population was treated with bDMARDs. By June 2018 , 20\% of the population used a bDMARD and $28 \%$ of those were treated with the ETN biosimilar.

Interrupted time series depicting the RMC in the total $R A$ population. The RMC of the total RA population in June 2014 was estimated at $€ 31,6521$ (95\% CI €29,746-€335,796, $P<0.001$ ) per quarter, and these costs appeared to increase significantly every quarter prior to June 2016 by $€ 19,982$ (95\% CI $€ 15,842-€ 24,121, P<0.001)$. In the first quarter after the price drop due to the introduction of the ETN biosimilar (June 2016), there appeared to be a significant decrease in RMC of $-€ 63,179$ (95\% CI -€97,638 to €28,718, $P=0.002$ ). However, the postinterruption cost trend did not change relatively to the pre-trend (€212, 95\% CI -€6629 to $€ 7054, P=0.947$ ) and total costs were back to the level at the moment of interruption after 3 quarters.
All absolute postinterruption trends are given in Supplementary Table 1 (available with the online version of this article).

How heavily the RMC is influenced by the cost for bDMARDs becomes apparent when studying the respective influence of bDMARDs, csDMARDs, and GCs on the RMC. The RMC of the total RA population adjusted for bDMARD cost (giving the cost of csDMARDs and GCs) was estimated at $€ 10,868$ (95\% CI $€ 4467-€ 17,269, P=0.003$ ). This means that the cost for bDMARDs accounted for approximately $96 \%$ of the total RMC. Interestingly, the average cost per patient for csDMARDs and GCs showed an increase over time. Since prices for these medications were assumed stable, this is an indication of intensifying treatment.

Interrupted time series depicting the RMC for biologic users. The total RMC of patients being treated with bDMARDs in June 2014 was estimated at $€ 301,250$ (95\% CI $€ 282,570-€ 319,930$, $P<0.001)$, and these appeared to increase significantly every quarter prior to June 2016 by $€ 19,242$ (95\% CI $€ 15,236-€ 23,248, P<0.001)$. In the first quarter after the price drop, there appeared to be a significant decrease in total RMC of -€63,020 (95\% CI -€96,487 to -€29,553, $P=0.001$ ), whereas the quarterly posttrend of total RMC (relative to the pre-price interruption trend) stayed more or less the same (€9, 95\% CI -€6695 to $€ 6715, P=0.998$; Figure $1 \mathrm{~A}$ ).

The average RMC per patient being treated with bDMARDs in June 2014 was estimated at $€ 2869$ (95\% CI €2727-€3011, P $<0.001)$ per quarter, and these appeared to increase significantly every quarter prior to the second quarter of 2016 by $€ 31$ (95\% CI $€ 2-€ 61, P=0.041)$. In the first quarter after the price decrease, there appeared to be a significant decrease in average RMC per patient of $-€ 370$ (95\% CI $-€ 602$ to $-€ 138, P=0.005$ ), followed by a significant decrease in the quarterly trend of the average RMC per patient (relative to the pre-price interruption trend) of $-€ 50.34$ per quarter (95\% CI $-€ 86$ to $-€ 14$, $P=0.010$; Figure 1B). Contrary to the total RMC curve, the average RMC curve for biologic users bends downward after the drop in price, implying that the average cost per patient using bDMARDs decreased further over time. Adjustment for the bDMARD dosage as a percent of the daily defined doses did not alter the average RMC per patient (-€682, 95\% CI

Table 1. Patient characteristics of the total population and the subgroup of patients using bDMARDs on June 1, 2016.

\begin{tabular}{lcccc}
\hline & \multicolumn{2}{c}{ RA Population, $\mathrm{n}=827$} & \multicolumn{2}{c}{ bDMARD Users, $\mathrm{n}=141$} \\
& Values & $\mathrm{n}$ & Values & $\mathrm{n}$ \\
\hline Age, yrs, mean (SD) & $63(14)$ & 827 & $58(14)$ & 141 \\
Disease duration, yrs, mean (SD) & $8(8)$ & 824 & $11(9)$ & 141 \\
Female sex, \% & 65 & 541 & 70 & 99 \\
DAS28, mean (SD) & $3.1(1.2)$ & 630 & 3.3 & $3.3(1.5)$ \\
RF positive, \% & 57 & $392 / 683$ & 56 & $69 / 123$ \\
Anti-CCP positive, \% & 55 & $378 / 684$ & 63 & $78 / 123$ \\
BMI, kg/m mean $(S D)^{2}$ m & $27(10)$ & 432 & $26(5)$ & 58 \\
\hline
\end{tabular}

Anti-CCP: anticyclic citrullinated peptide antibody; bDMARD: biologic disease-modifying antirheumatic drug; DAS28: Disease Activity Score in 28 joints; RA: rheumatoid arthritis; RF: rheumatoid factor. 


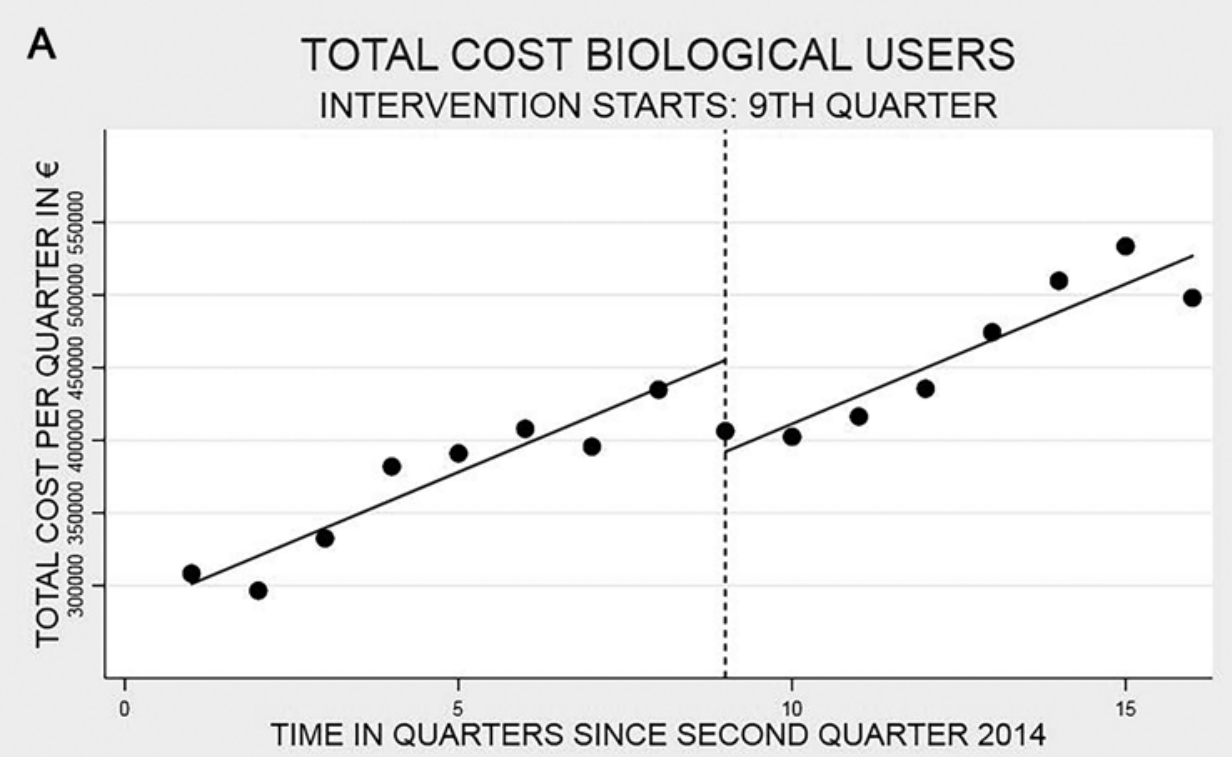

- actual PREDICTED

REGRESSION WITH NEWEY-WEST STANDARD ERRORS - LAG(2)

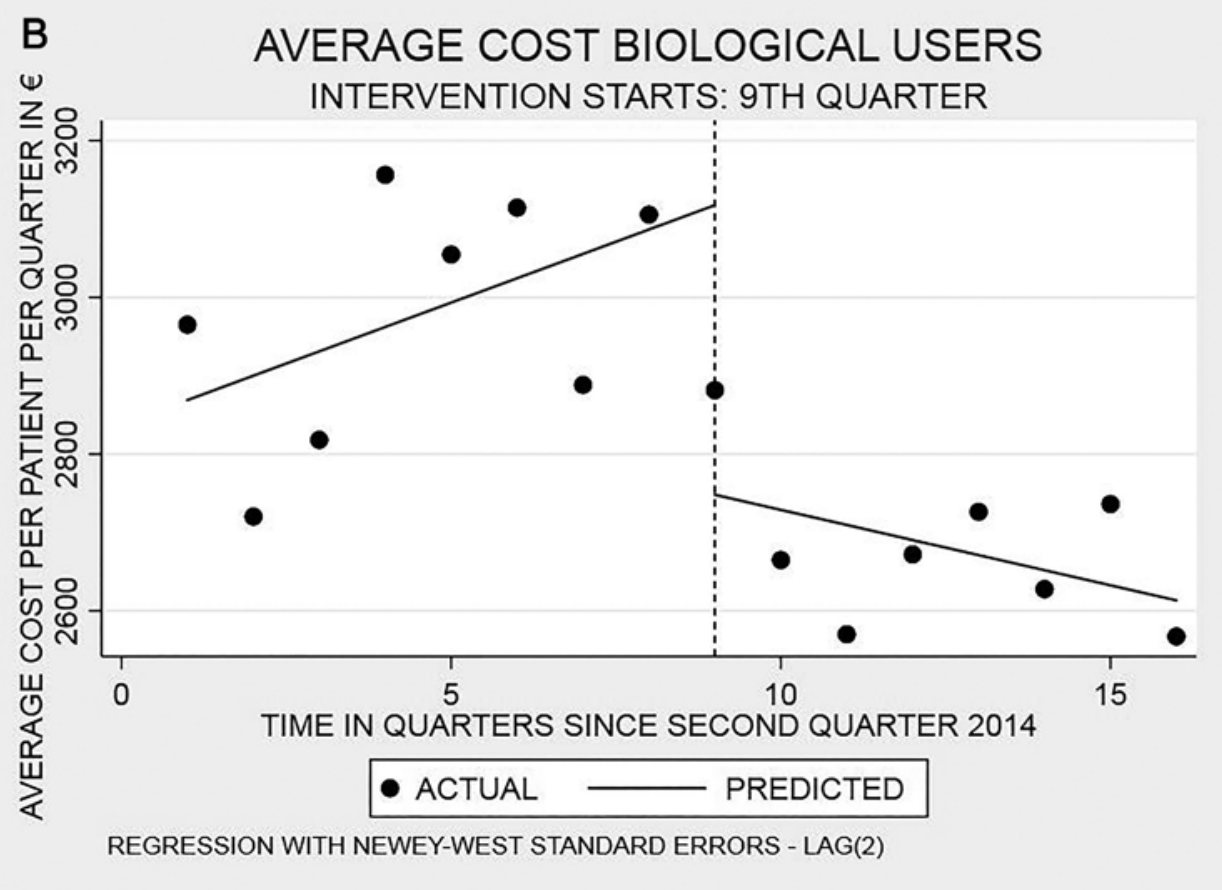

Figure 1. bDMARD cost and usage from June 1, 2014, to June 1, 2018. (A) Total medication cost of patients being treated with bDMARDs. (B) Average cost per patient being treated with bDMARDs. bDMARD: biologic disease-modifying antirheumatic drug.

$-€ 4860$ to $€ 3497, P=0.726)$. This means that there was no effect of dose intensity on the average cost per patient for biologic rheumatic medication.

This seeming paradox that the curve for total RMC in bDMARD users does not bend, whereas the average cost per bDMARD user drops can be explained by looking at the number of biologic users in the total RA population. There was a significant increase $(0.22 \%, 95 \%$ CI $0.11-0.32, P=0.001)$ per quarter in percentage of patients being treated with bDMARDs prior to the introduction of biosimilars (Figure 1C). This trend significantly increased further after the introduction of the ETN biosimilar $(0.28 \%, 95 \%$ CI $0.10-0.47, P=0.006)$, leading to a significant posttrend increase per quarter $(0.50 \%, 95 \% \mathrm{CI}$ $0.3791-0.6204, P<0.001$; Figure $1 \mathrm{C})$. This increase in patients 


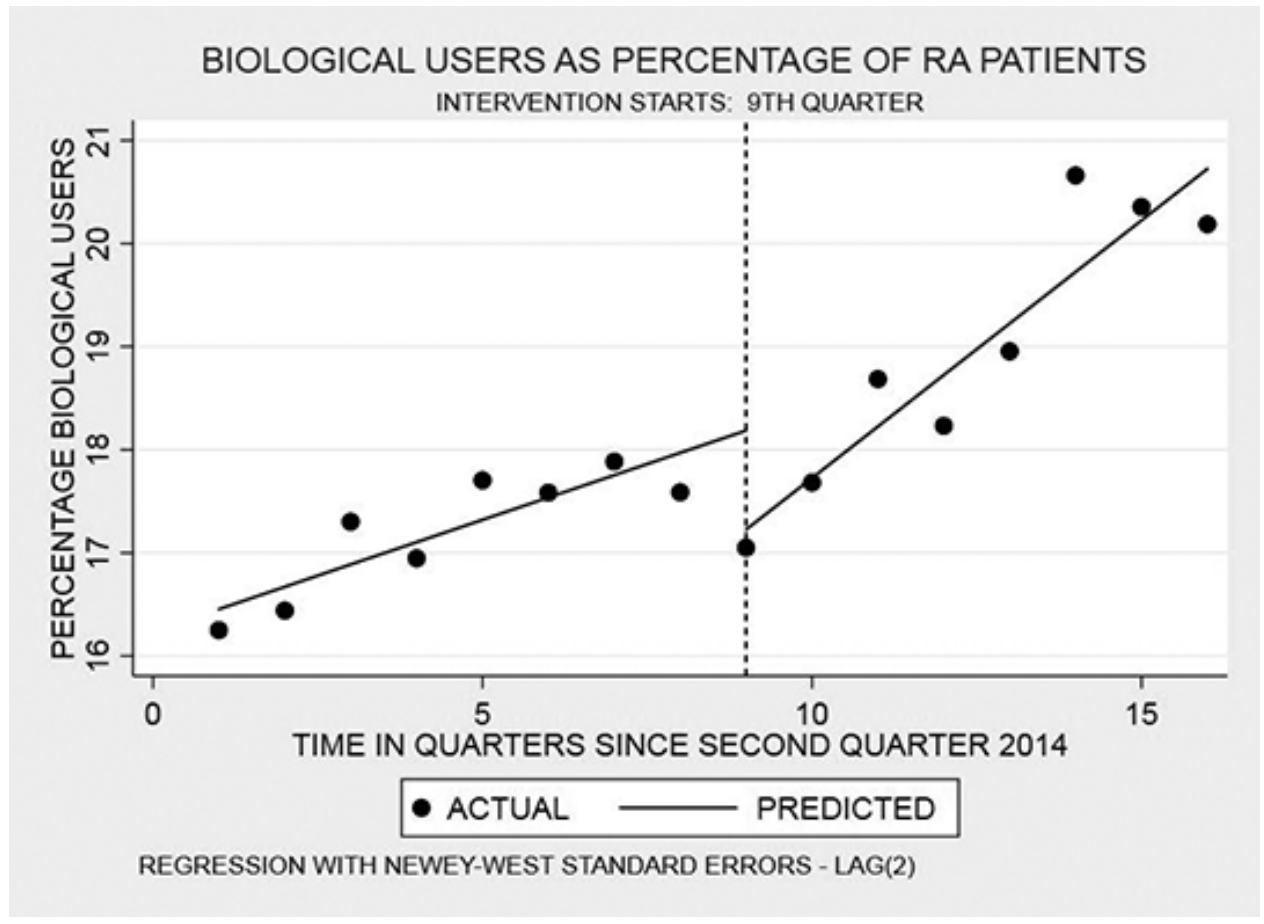

Figure 1. (C) The percentage of patients who were bDMARD users. bDMARD: biologic disease-modifying antirheumatic drug.

being treated with bDMARDs counterbalances the individual price reduction achieved by biosimilars.

Can trends in cost be explained by demographics or disease-specific variables of biologics users over time? Table 2 shows the patient characteristics and medication use of patients initiating their first biologic treatment before and after the introduction of the ETN biosimilar. After the introduction of the biosimilar, patients tend to be older at the initiation of their first bDMARD and use fewer csDMARDs as co-medication. To study the influence of changes in patient characteristics, 2 adjusted models (mentioned in the Methods section) were run. Both the inclusion of the demographic variables (adjusted model I) and inclusion of the RA disease-specific variables (adjusted model II) could not explain the cost results in a significant way (Supplementary Tables 2 and 3 , available with the online version of this article). However, the variable patients' age at initiation of first biologic was near significant $(P=0.057)$, meaning that bDMARD users in the post-interruption period were increasingly older.

\section{DISCUSSION}

This study observes the bending of the average medication cost curve for patients being treated with a bDMARD when an ETN biosimilar becomes available. However, we notice that the trend in the total cost curve stays the same, (i.e., no sustainable free disposable savings could be collected). This occurs because price reduction achieved by the introduction of the biosimilar facilitates an increase in bDMARD users, which counteracts the initial cost savings.

This study was applied in a real-world setting. A strength of this approach is that the data provide the opportunity to study which patients gained access to bDMARDs. Next to that, the interrupted time series design has the capability of identifying underlying trends, thereby isolating the effect of the introduction of biosimilars on the trend. This increases the confidence with which observed effects can be attributed to the introduction of biosimilars. A limitation is that data from only 1 hospital were obtained for analysis. However, Bernhoven is a typical Dutch referral center, with a case mix of patients that is representative for general hospitals in the Netherlands with respect to its RA population. We verified this by comparing our population with the RA population from the DREAM consortium, a collaboration between 13 hospitals in the Netherlands. Our RA population and the subgroup of bDMARD users were comparable on age, sex, disease duration, and rheumatic antibody levels. ${ }^{19}$ Findings regarding increased utilization were also in line with other (nationwide) real-world data, further strengthening our conclusions. ${ }^{1,11}$

After the initial price reduction achieved through the market entry of the ETN biosimilar, we observed a bending in average medication cost curve for bDMARD users. This means that the average treatment cost per patient for bDMARD users decreased over time after the availability of the ETN biosimilar. This corresponds to the gradual increase in uptake of the ETN biosimilar, driving down the cost. At the same time, the total medication cost curve did not change, because the potential savings achieved through biosimilar use were used to further increase prescription of bDMARDs. These findings are similar to the NZa report, which shows that across different indications, the volume of patients using expensive medication increases, while the budget remains more or less stable. ${ }^{1}$

In Bernhoven, the additional increase in bDMARD prescription after the introduction of the biosimilar happened 
Table 2. Patient characteristics and medication use of patients starting biologic treatment in the years 2015 and 2017.

\begin{tabular}{|c|c|c|c|c|}
\hline & \multicolumn{2}{|c|}{$\begin{array}{r}\text { Before Introduction } \\
\text { of Biosimilar, } n=59 \\
N\end{array}$} & \multicolumn{2}{|c|}{$\begin{array}{r}\text { After Introduction } \\
\text { of Biosimilar, } n=67 \\
N\end{array}$} \\
\hline \multicolumn{5}{|l|}{ Baseline characteristics } \\
\hline Mean (SD) age at biologic initiation, yrs & $52(14)$ & 59 & $58(14)$ & 67 \\
\hline Mean (SD) disease duration at biologic initiation, yrs & $4(6)$ & 59 & $4(6)$ & 67 \\
\hline Female sex, \% & 74.6 & 44 & 70.1 & 47 \\
\hline RF positive, $\%$ & 42.3 & $22 / 52$ & 56.4 & $22 / 39$ \\
\hline Anti-CCP positive, $\%$ & 51.9 & $27 / 52$ & 55.6 & $25 / 45$ \\
\hline $\mathrm{BMI}$, mean $(\mathrm{SD})$ & $27.0(7)$ & 40 & $27.8(7)$ & 35 \\
\hline DAS28 at biologic initiation, mean (SD) & $4.7(1.3)$ & 38 & $4.5(1.2)$ & 42 \\
\hline \multicolumn{5}{|l|}{ Medication use prior to initiation of first bDMARD } \\
\hline No. csDMARDs used, mean (SD) & $2.1(0.7)$ & 57 & $2.1(0.7)$ & 60 \\
\hline Patients with GC treatment in last year, $\%$ & 73 & 43 & 73 & 49 \\
\hline No. prednisone prescriptions in last year, mean (SD) & $2.7(2.5)$ & 59 & $2.6(2.6)$ & 67 \\
\hline \multicolumn{5}{|l|}{ Medication use during initiation of first bDMARD } \\
\hline \multicolumn{5}{|l|}{$\begin{array}{l}\text { Distribution of bDMARD utilization at initiation } \\
\text { of first bDMARD }\end{array}$} \\
\hline Abatacept & - & - & 2 & 1 \\
\hline Adalimumab & 53 & 31 & 42 & 28 \\
\hline Etanercept & 36 & 21 & 46 & 31 \\
\hline Certolizumab & - & - & 3 & 2 \\
\hline Golimumab & 3 & 2 & 2 & 1 \\
\hline Infliximab & 2 & 1 & - & - \\
\hline Rituximab & 5 & 3 & 3 & 2 \\
\hline Tocilizumab & 2 & 1 & 3 & 2 \\
\hline \multicolumn{5}{|l|}{ No. csDMARD at biologic initiation (co-medication) } \\
\hline 0 & 14 & 8 & 27 & 18 \\
\hline 1 & 44 & 26 & 37 & 25 \\
\hline 2 & 42 & 25 & 35 & 24 \\
\hline Patients using MTX at biologic initiation, \% & 68 & 40 & 54 & 36 \\
\hline
\end{tabular}

Anti-CCP: anticyclic citrullinated peptide antibody; bDMARD: biologic disease-modifying antirheumatic drug; csDMARD: conventional synthetic disease-modifying antirheumatic drug; DAS28: Disease Activity Score in 28 joints; GC: glucocorticoid; MTX: methotrexate; RF: rheumatoid factor.

unconsciously and autonomously. The rheumatologists had not consciously changed their prescription policy, and only became aware of the increase in bDMARD prescription after the conducting of this research. The question arises whether this automatic return of savings to RA care, where it funds an increase in bDMARD prescriptions is desirable. Where current literature focuses only on national declaration data and lacks patient-specific demographic and medical data, ${ }^{1,11}$ our data offer the possibility to assess which patients gained access to bDMARD therapy. It is known that older patients are lesslikely to receive biologic treatment. ${ }^{20,21}$ The observed increasing prescription of bDMARDs could be a response to previously undertreatment in that group. The interrupted time series (adjustment model II) shows that bDMARD users tend to be older after the introduction of biosimilars, supporting this hypothesis. The adjustment models were unable to detect other differences between the pre- and postinterruption group. This was perhaps due to insufficient power to detect differences at a group level. When focusing on patients initiating bDMARD therapy, we observed that after the introduction of the biosimilar, patients were using less csDMARD co-medication at the initiation of
bDMARD therapy. The percent of patients using methotrexate as co-medication dropped from $68 \%$ to $54 \%$. This could be an indication that patients were given the chance, by initiating a bDMARD, to stop their csDMARD, which is known to have adverse effects.

The actual health benefits of additional bDMARD prescriptions remain very difficult to assess. On a population level, there was no change in disease activity during the study period, but we observed a small nonsignificant improvement in disability (data not shown). However, in absolute numbers, there was only a small increase in bDMARD users (i.e., $4 \%$ ). Therefore, possible effects are diluted on a population level and difficult to assess. In relative numbers, there was an increase of nearly $25 \%$ in the number of bDMARD users. A change in the type of patients receiving bDMARD therapy comes with the risk that the treatment is less effective in the new target population. ${ }^{22,23}$ Expansion of bDMARD therapy to older patients might affect the cost-benefit ratio of bDMARD therapy and should be further examined. A previous study showed that increased access to bDMARDs might not be the best investment from a societal perspective. ${ }^{22}$ This study reported that reinvesting biosimilar savings in RA 
care came in only at fifth place in cost-effectiveness if quality of life maximization is feasible, in a country where patients have ready access to bDMARDs. ${ }^{22}$

At this point, the general opinion is that biosimilars have the potential to generate billions of euros in savings in Europe alone, ${ }^{24}$ and that payers are likely to experience some relief of budgetary constraints or the ability to reallocate funds, depending on the policy priorities of each country. ${ }^{22}$ Already, biosimilars help reduce access inequities and lead to an increase in bDMARD prescription in Europe. ${ }^{25}$ We found that the total medication cost before and after the introduction of biosimilars remained more or less the same, whereas the number of bDMARD users increased. Therefore, the assumption that the availability of biosimilars facilitates increased access to biologic therapy in Europe seems valid. ${ }^{24}$ However, the discrepancy between expected and realized budgetary saving is significant. Our study and other available data regarding real-world savings show that no net savings were achieved by biosimilar use, because freed up funds were used for increasing access to biologic therapy within the same indication. ${ }^{1,11}$ This phenomenon - that a price decrease leads to an increase in volume of patients treated-is well known in economics but is often overlooked and seldom anticipated in real-world policy making. ${ }^{26}$ Further research should focus on the cost-effectiveness of expanding access to bDMARD therapy.

\section{ONLINE SUPPLEMENT}

Supplementary material accompanies the online version of this article.

\section{REFERENCES}

1. Dutch Healthcare Authority. Monitor medicines in specialist medical care January 2019. [Internet. Accessed March 31, 2021.] Available from: puc.overheid.nl/nza/doc/PUC_264248_22/1

2. World Health Organization. Guidelines on evaluation of similar biotherapeutic products (SBPs). [Internet. Accessed April 5, 2021.] Available from: www.who.int/biologicals/areas/biological_therapeutics/ BIOTHERAPEUTICS_FOR_WEB_22APRIL2010.pdf

3. Brodszky V, Rencz F, Péntek M, Baji P, Lakatos PL, Gulácsi L. A budget impact model for biosimilar infliximab in Crohn's disease in Bulgaria, the Czech Republic, Hungary, Poland, Romania, and Slovakia. Expert Rev Pharmacoecon Outcomes Res 2016;16:119-25.

4. Mulcahy AW, Predmore Z, Mattke S. The cost savings potential of biosimilar drugs in the United States. 2014. [Internet. Accessed March 31, 2021.] Available from: www.rand.org/pubs/perspectives/ PE127.html

5. Gulácsi L, Brodszky V, Baji P, Rencz F, Péntek M. The rituximab biosimilar CT-P10 in rheumatology and cancer: a budget impact analysis in 28 European countries. Adv Ther 2017;34:1128-44.

6. Aladul MI, Fitzpatrick RW, Chapman SR. The effect of new biosimilars in rheumatology and gastroenterology specialities on UK healthcare budgets: results of a budget impact analysis. Res Social Adm Pharm 2019;15:310-7.

7. Hutton D, Newman-Casey PA, Tavag M, Zacks D, Stein J. Switching to less expensive blindness drug could save medicare part B \$18 billion over a ten-year period. Health Aff 2014;33:931-9.

8. Brodszky V, Baji P, Balogh O, Péntek M. Budget impact analysis of biosimilar infliximab (CT-P13) for the treatment of rheumatoid arthritis in six central and eastern European countries. Eur J Health Econ 2014;15 Suppl 1:S65-71.

9. Generics and Biosimilars Initiative. Biosimilars approved in Europe. 2011. [Internet. Accessed March 31, 2021.] Available from: www.
gabionline.net/Biosimilars/General/Biosimilars-approved-in-Europe

10. Heal A. NHS replaces highest-spend drug with $£ 300 \mathrm{~m}$ cheaper alternative. [Internet. Accessed March 31, 2021.] Available from: www.theguardian.com/society/2018/nov/26/nhs-replaces-highestspend-drug-with-300m-cheaper-alternative

11. Aladul MI, Fitzpatrick RW, Chapman SR. Impact of infliximab and etanercept biosimilars on biological disease-modifying antirheumatic drugs utilisation and NHS budget in the UK. BioDrugs 2017;31:533-44.

12. Voorneveld-Nieuwenhuis JE. Herziene richtlijn biologicals December 2010. [Article in Dutch] Nederlands Tijdschrift voor Reumatologie 2010;4.

13. Müskens WD, Rongen-van Dartel SAA, Adang EMM, van Riel PL. Biosimilars in the management of chronic inflammatory diseases: the Dutch experience. Mediterr J Rheumatol 2019;30 Supp 1:76-81.

14. Aletaha D, Neogi T, Silman AJ, Funovits J, Felson DT, Bingham CO III, et al. 2010 rheumatoid arthritis classification criteria: an American College of Rheumatology/European League Against Rheumatism collaborative initiative. Ann Rheum Dis 2010;69:1580-8.

15. Prevoo ML, van ' $t$ Hof MA, Kuper HH, van Leeuwen MA, van de Putte LB, van Riel PL. Modified disease activity scores that include twenty-eight-joint counts. Development and validation in a prospective longitudinal study of patients with rheumatoid arthritis. Arthritis Rheum 1995;38:44-8.

16. National Health Care Institute. Cost guide. Cost research methodology and reference prices for economic evaluations in health care. 2015 [Internet. Accessed March 31, 2021.] Available from: www.zorginstituutnederland.nl/publicaties/ publicatie/2016/02/29/richtlijn-voor-het-uitvoeren-vaneconomische-evaluaties-in-de-gezondheidszorg

17. Linden A, Adams JL. Applying a propensity score-based weighting model to interrupted time series data: improving causal inference in programme evaluation. J Eval Clin Pract 2011;17:1231-8.

18. WHO Collaborating Centre for Drug Statistics Methodology. Antineoplastic and immunomodulating agents. 2020. [Internet. Accessed March 31, 2021.] Available from: www.whocc.no/ atc_ddd_index $/$ ?code $=$ L\&showdescription $=$ no

19. DREAM. [Transparency and efficiency in rheumatism care 2017.] [Internet. Accessed April 5, 2021.] Available from: www. dreamregistry.nl/uploads/media/5bb1f9e4ffffd/DREAM\%20 rapport\%202017_final.pdf

20. Morsley K, Kilner T, Steuer A. Biologics prescribing for rheumatoid arthritis in older patients: A single-center retrospective cross-sectional study. Rheumatol Ther 2015;2:165-72.

21. Tutuncu Z, Reed G, Kremer J, Kavanaugh A. Do patients with older-onset rheumatoid arthritis receive less aggressive treatment? Ann Rheum Dis 2006;65:1226-9.

22. Martin M, Campbell R, Jacob J. Reinvestment of biosimilar savings: What are the best options? [abstract]. Ann Rheum Dis 2018;77 Suppl 2.

23. Fuchs VR. More variation in use of care, more flat-of-the-curve medicine. Health Aff 2004; Suppl variation:VAR104-7.

24. IMS Institute for Healthcare Informatics. Delivering on the potential of biosimilar medicines: the role of functioning competitive markets. [Internet. Accessed April 5, 2021.] Available from: www.medicinesforeurope.com/wp-content/ uploads/2016/03/IMS-Institute-Biosimilar-Report-March-2016FINAL.pdf

25. Baumgart DC, Misery L, Naeyaert S, Taylor PC. Biological therapies in immune-mediated inflammatory diseases: can biosimilars reduce access inequities? Front Pharmacol 2019;10:279.

26. Salm M, Wübker A. Do hospitals respond to decreasing prices by supplying more services? Health Econ 2020;29:209-22. 